\title{
Portal Vein Thrombosis in a Patient with Hereditary Antithrombin Deficiency
}

\author{
Tamao Setaka ${ }^{1}$, Katsuharu Hirano ${ }^{1}$, Keiichi Moriya ${ }^{1}$, Tougen Kaneko ${ }^{1}$, Seie Morita ${ }^{1}$, \\ Tetsu Shinkai ${ }^{1}$, Eriko Morishita ${ }^{2}$ and Takafumi Ichida ${ }^{1}$
}

\begin{abstract}
:
Portal vein thrombosis (PVT) has been reported in many patients with and without liver cirrhosis. The portal vein is a rare site of thrombosis, and various conditions can predispose an individual to PVT. Among those conditions, hereditary thrombophilia has been increasingly reported recently. We herein report the case of a non-cirrhotic 30-year-old man who developed acute PVT with hereditary antithrombin deficiency. Antithrombin (AT) replacement therapy was required along with heparin. Given our experience with this case, we believe that a screening test for prothrombotic disorders, such as AT deficiency, should be considered in cases of PVT.
\end{abstract}

Key words: portal vein thrombosis, antithrombin deficiency, anticoagulation therapy

(Intern Med 58: 1733-1737, 2019)

(DOI: 10.2169/internalmedicine.2295-18)

\section{Introduction}

Portal vein thrombosis (PVT) refers to the condition in which a thrombus develops in the trunk of the portal vein or the right and left intrahepatic portal branches. Balfour and Stewart reported the first case of PVT in 1868 in a patient with ascites, splenomegaly, and variceal dilation (1). Since then, PVT has been well studied as a complication of liver diseases, but it has also been reported in patients with or without liver diseases. The prevalence of PVT is reported to be $0.6-16 \%$ in patients with compensated cirrhosis and $35 \%$ in patients with decompensated cirrhosis (2-4). The lifetime risk of PVT in the general population is $1 \%$ (5). The pathophysiology of PVT can be explained by Virchow's triad: a procoagulant state, reduced portal flow, and endothelial injury of the portal vein. For example, cirrhosis is a wellknown risk factor of PVT. Portal hypertension is a main complication of cirrhosis that causes turbulent portal flow, resulting in a reduced velocity of the portal flow. In addition, coagulant factors and anticoagulant factors are present at low levels in cirrhosis patients, but the opposite can easily occur. Other risk factors include abdominal inflammation, malignancy, surgery, trauma, and pregnancy. In recent studies, prothrombotic disorders, such as myeloproliferative disorders, paroxysmal nocturnal hemoglobinuria, antiphospholipid syndrome, hyperhomocysteinemia, protein C deficiency, protein $\mathrm{S}$ deficiency, factor $\mathrm{V}$ Leiden mutation, and antithrombin deficiency, have been reported to be associated with the development of PVT.

We herein report a case of PVT in a patient with hereditary antithrombin (AT) deficiency.

\section{Case Report}

The patient's privacy is protected because no personal data are included in the manuscript.

A 30-year-old Japanese man presented to the emergency department with the sudden onset of abdominal pain. He had no remarkable medical history. His family history was unremarkable for any thrombotic event. A physical examination revealed a fever of $38^{\circ} \mathrm{C}$ and slight tenderness in the epigastric region. Results of the laboratory analysis revealed a white blood cell count of $14.1 / \mathrm{mm}^{3}$ and C-reactive protein level of $9.72 \mathrm{mg} / \mathrm{dL}$ (normal range, $<0.8 \mathrm{mg} / \mathrm{dL}$ ). The fibrin degradation product level was elevated to $36.2 \mu \mathrm{g} / \mathrm{L}$ (normal

${ }^{1}$ Department of Internal Medicine, Shonan East General Hospital, Japan and ${ }^{2}$ Department of Clinical Laboratory Sciences, Graduate School of Medical Science, Kanazawa University, Japan

Received: October 24, 2018; Accepted: December 4, 2018; Advance Publication by J-STAGE: February 25, 2019

Correspondence to Dr. Katsuharu Hirano, khirano-tym@umin.ac.jp 


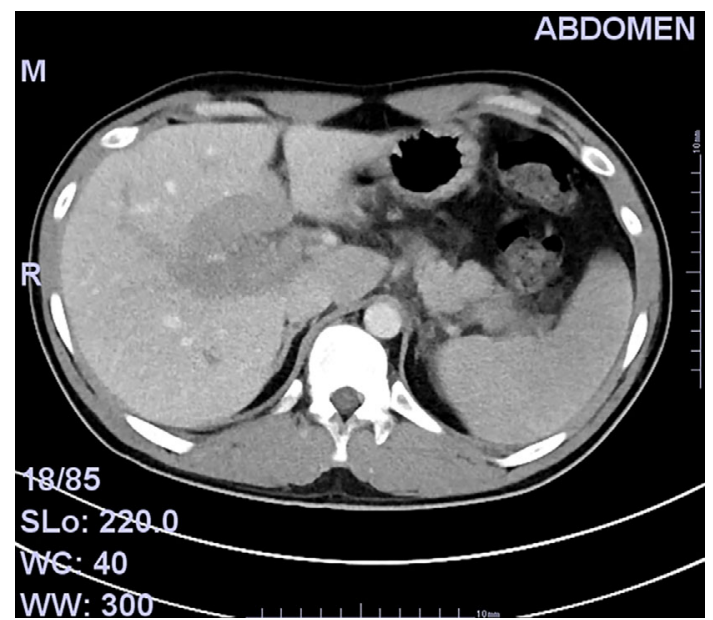

Figure 1. Contrast-enhanced CT on the second hospital day demonstrating the dilated portal vein filled with a thrombus.

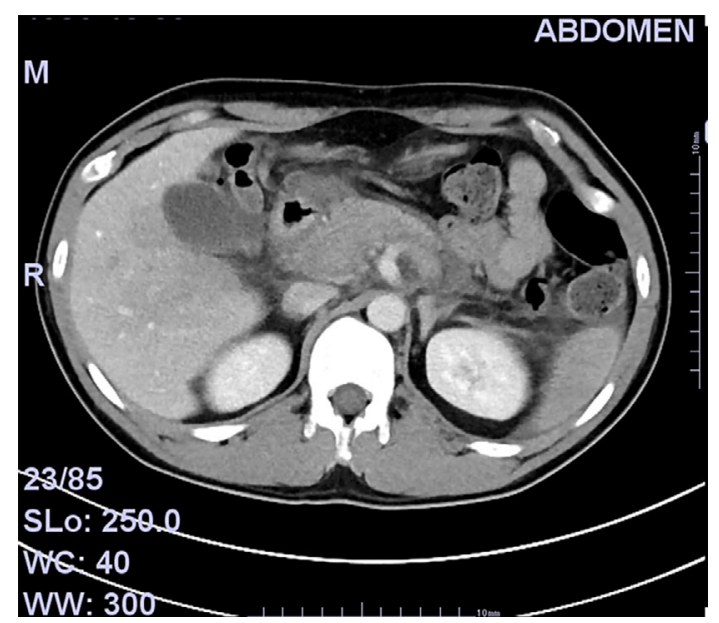

Figure 3. Inflammatory fat stranding around the pancreatic head.

range, $<10 \mu \mathrm{g} / \mathrm{mL}$ ), which was indicative of a thrombus. The D-dimer level was not examined at admission. Upper gastrointestinal endoscopy and non-contrast computed tomography (CT) did not show any abnormal changes. Ultrasonography at admission did not show any typical abnormality, including changes such as a dilated portal vein. Doppler ultrasonography was not performed since we did not suspect PVT at this point. On the second hospital day, contrastenhanced CT was performed, which demonstrated a filling defect in the dilated portal vein, indicating the presence of a thrombus (Fig. 1, 2). The thrombus obstructed the vessel lumen of the main trunk of the portal vein and the superior mesenteric vein (Fig. 1). In addition, it revealed inflammatory fat stranding around the pancreatic head (Fig. 2, 3). We diagnosed him with PVT and started anticoagulation therapy.

Since an association between thrombophilia and PVT has been reported in many cases $(6,7)$, we additionally conducted a screening test for prothrombotic disorders to identify the underlying condition that could have predisposed

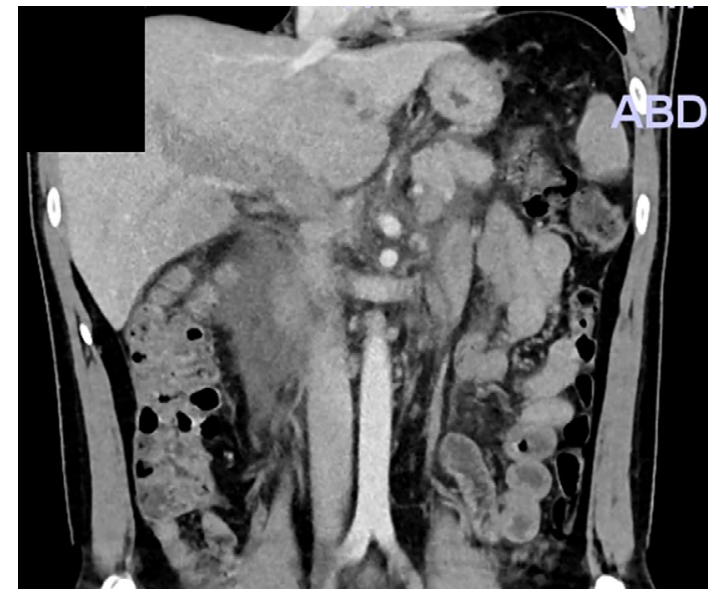

Figure 2. Contrast-enhanced CT on the second hospital day demonstrating the dilated portal vein filled with a thrombus and the inflammatory fat stranding around the pancreatic head.

this patient to PVT. The result showed that the AT activity was decreased at 56\%. Protein $\mathrm{C}$ activity, protein S activity, anticardiolipin antibody, lupus anticoagulant, lipoprotein(a), and anticardiolipin $\beta 2$-glycoprotein I antibody levels were all within normal ranges. Hereditary AT deficiency was suspected, so we measured the AT activity of the patient's relatives. Only the father's AT activity was decreased at $49.5 \%$, although he had no history of thrombosis.

Under the diagnosis of AT deficiency, intravenous heparin was started on hospital day 6. Along with heparin, we administered intravenous AT (1,500 U/day) from hospital days 12 to 14 . We switched heparin to oral warfarin from hospital day 16 , but the prothrombin time-international normalized ratio (PT-INR) decreased to 1.09 on hospital day 22, which was below the therapeutic range. In addition, the D-dimer level, which was first measured on day 19, was increased at $24.9 \mu \mathrm{g} / \mathrm{mL}$ (normal range, $<0.5 \mu \mathrm{g} / \mathrm{mL}$ ), so we re-started heparin on hospital day 22 and added AT again from hospital days 24 to 25 . The $\mathrm{D}$-dimer level then decreased from $21.7 \mu \mathrm{g} / \mathrm{mL}$ (hospital day 21) to $3.8 \mu \mathrm{g} / \mathrm{mL}$ (hospital day 26). Heparin was completely switched to oral warfarin on hospital day 29. Contrast-enhanced CT on hospital day 16 also demonstrated regression of the thrombus and inflammatory fat stranding around the pancreatic head (Fig. 4).

The patient was discharged on hospital day 33 with oral warfarin. The PT-INR was well controlled within the target range (1.5-2.5). However, the thrombus in the portal vein remained. Five months later, CT showed cavernous transformation of the portal vein (Fig. 5), and upper gastrointestinal endoscopy revealed a gastric varix. These findings can be explained as complications of portal hypertension due to chronic obstruction of the portal vein.

Along with the treatment of PVT, a direct DNA sequencing analysis of the SERPINCl gene, which encodes AT, was conducted. The point mutation c.1314C $>$ A (p.Pro439Thr) was identified. A polymerase chain reaction-restriction fragment length polymorphism analysis also revealed the same 


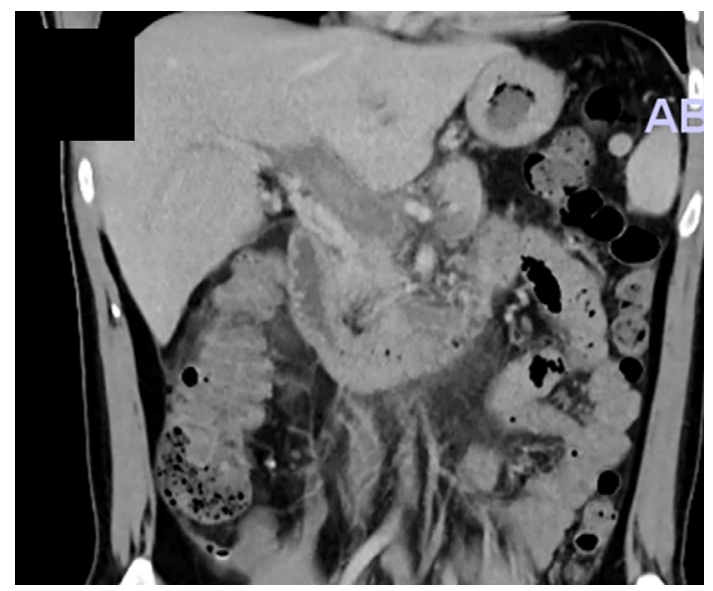

Figure 4. Contrast-enhanced CT on hospital day 16 demonstrating regression of the thrombus and inflammatory fat stranding around the pancreatic head.

mutation in the patient's father. This mutation was reported as AT Budapest, a type II-pulmonary embolism variant AT deficiency.

\section{Discussion}

Heterozygous AT deficiency is an autosomal dominant disease that occurs in $0.02-0.17 \%$ of the general population and in $0.5-4.9 \%$ of patients with venous thromboembolism (VTE) (8-12). AT is an important physiological inhibitor of activated serine proteinases of the coagulation system, such as thrombin. The formation of complexes of thrombin and AT is accelerated at least 2000-fold in the presence of heparin, and a similar enhancement is seen for the inhibition of factors Xa and IXa (13). In vivo, this function is subserved by glycosaminoglycan sulfate localized on endothelial cell surfaces (14).

The diagnosis and classification of AT deficiency require antithrombin assays. There are two types of antithrombin assays: activity and antigen assays. Activity assays should be performed for the initial screening of AT deficiency, as antigen levels are often normal in subtypes with functional defects of AT. Antigen assays are immunoassays designed to measure the quantity of protein regardless of the protein's ability to function (15). Because of the presence of interlaboratory variations, most laboratories express AT antigen and activity levels in terms of percentages, with normal ranges being approximately $80-120 \%$ (16). Most patients with inherited, heterozygous AT deficiency have AT activity levels in the range of $40-60 \%$ (16). If the AT activity is decreased, an antigen assay should be considered in order to determine the subtype. Type I deficiencies are characterized by decreased AT activity and antigen levels. Type II deficiencies are characterized by decreased activity and normal antigen levels due to the decreased function of AT. Type II deficiencies are further divided into three types: reactive site, heparin binding site, and pleiotropic effects.

The main clinical features of thrombosis with AT defi-

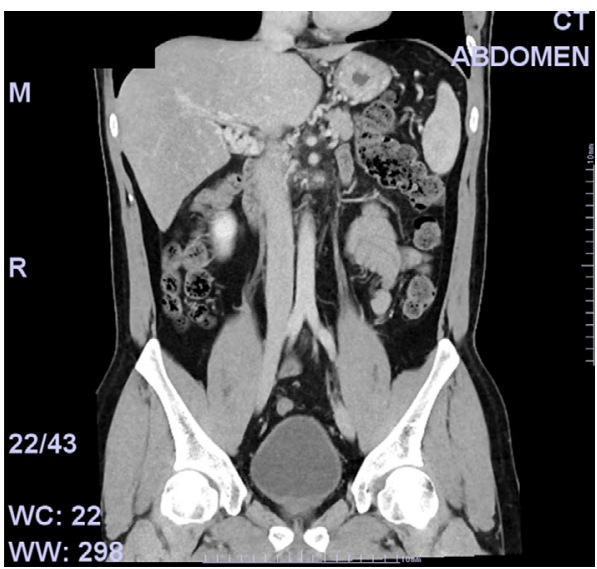

Figure 5. Contrast-enhanced CT five months after discharge showing cavernous transformation of the portal vein.

ciency are onset at a young age, idiopathic thrombosis, a family history of thrombosis, and recurrent VTE. Between $30 \%$ and $80 \%$ of carriers have thrombosis, and the risk is highest in those between 15 and 30 years of age (17). The most frequent thrombosis in AT deficiency is deep venous thrombosis, but thrombosis in rare sites, such as the inferior vena cava, mesenteric vein, and sagittal sinus, has also been reported (18).

For the treatment of thrombosis with AT deficiency in the acute phase, AT replacement therapy along with heparin might be required because individuals with AT deficiency may have heparin resistance, since heparin acts dependently on AT. AT replacement therapy has been reported to successfully achieve regression of the thrombus in some cases of thrombosis with AT deficiency (19-21). However, no randomized clinical trials have assessed the efficacy of AT replacement therapy. After the administration of heparin and AT replacement therapy, given the high risk of recurrent venous thromboembolism in AT deficiency (22), prophylactic anticoagulation therapy should be continued. Warfarin has long been used for prophylaxis, effectively reducing the recurrence risk (22).

Another choice for prophylaxis of recurrent thrombosis in AT deficiency is direct oral anticoagulants (DOACs). Theoretically, DOACs directly block the activity of coagulant factors IIa and Xa, unlike heparin, by increasing the activity of AT. We can therefore expect DOACs to be a good option for thrombosis with AT deficiency. An animal experiment suggested that edoxaban might be effective in individuals with low AT concentrations (23). The efficacy of DOACs for AT thrombosis with AT deficiency has been described in some case reports. Minami et al. reported a case of venous thromboembolism with AT deficiency treated by the factor Xa inhibitor rivaroxaban (24). Kawano et al. reported a case of deep vein thrombosis and pulmonary thromboembolism in a patient with AT deficiency successfully treated by edoxaban, another direct oral factor Xa inhibitor (25). However, to our knowledge, no clinical trials have assessed the efficacy of DOACs in cases of thrombosis with AT deficiency. 
We experienced a case of PVT in a 30-year-old man with AT deficiency. Although it was his first thrombotic event and his family history was unremarkable for thrombosis, hereditary thrombophilia and other predisposing factors were suspected because of the young age of onset (30 years) and unusual site of thrombus (portal vein). No typical episode could have led to this prothrombic state, such as trauma, surgical procedures, prolonged bed rest, or a long flight. However, the patient had visited a clinic complaining of abdominal pain 3 times in the 18 days before his admission. In this case, the recurrent abdominal pain may have been attributed to the abdominal inflammation that predisposed this patient to the formation of thrombus, along with his underlying AT deficiency. However, PVT itself can cause abdominal pain due to bowel congestion and ischemia, especially when the thrombus extends to the mesenteric vein. In the present case, contrast-enhanced CT showed that the thrombus had formed in the superior mesenteric vein as well as the portal vein, so the patient's bowel congestion may have been the cause of his abdominal pain. The inflammatory fat stranding around the pancreatic head can also be interpreted as indicative of either the existence of abdominal inflammation, such as acute pancreatitis, or the ischemic effect of the PVT.

The screening test of major thrombophilia showed a decreased AT activity at 56\%. According to previous studies, the normal range is $80-120 \%$ (16), and individuals with heterozygous inherited AT deficiency have an AT activity in the range of $40-60 \%$ (16). With the patient's AT activity level being in the lower range, hereditary AT deficiency was suspected. We tested the AT activity levels of the patient's parents and sister, and the father's AT activity was found to be decreased as well, which was consistent with our suspicion that the patient had hereditary AT deficiency. Therefore, a DNA analysis was performed to determine whether or not this was a case of hereditary AT deficiency from an AT gene mutation. The DNA analysis identified the gene mutation as AT Budapest. This type II-PE AT variant was first described in 1992 (26) and was reported in the Japanese population in 2009 (27). This p.Pro439Thr missense mutation is categorized as pleiotropic due to alterations in the reactive site and its heparin-binding properties (26). To treat the present patient, we administered AT concentrate along with heparin as recommended by previous studies and achieved regression of the thrombus. We also changed heparin to oral warfarin after the acute phase. As mentioned above, because thrombosis in patients with AT deficiency has a high recurrence rate (22), this patient will be encouraged to continue longterm prophylactic anticoagulation therapy. Although we started prophylactic anticoagulation with warfarin, researchers have reported cases that were successfully managed with DOACs in recent years $(24,25)$, so DOACs might be a good alternative treatment in the future.

Of additional note, the formation of collateral circulation was detected in the present case due to the long-term portal vein obstruction caused by the remaining thrombus; thus, regular follow-up of portal hypertension and management of its complications, such as esophageal varix, should be continued. Furthermore, the patient's father also had AT deficiency. He had no history of thrombosis, so he does not require anticoagulation therapy at present. However, AT replacement therapy should be considered if he undergoes surgery or sustains a traumatic injury in the future in order to prevent thrombosis.

\section{Conclusions}

The portal vein is a rare site of thrombosis with various underlying causes. One of the predisposing factors for PVT is prothrombotic disorders. We experienced a case of PVT in a young patient with AT deficiency, and AT replacement therapy was required along with heparin. Given our experience with this case, we believe that a screening test for prothrombotic disorders, such as AT deficiency, should be considered in cases of PVT.

\section{The authors state that they have no Conflict of Interest (COI).}

\section{Financial Support}

This research was partially supported by the Ministry of Health, Labour and Welfare.

\section{References}

1. Balfour GW, Stewart TG. Cases of enlarged spleen complicated with ascites, both depending upon varicose dilatation and thrombosis of the portal vein. Edinb Med J 14: 589-598, 1869.

2. Okuda K, Ohnishi K, Kimura K, et al. Incidence of portal vein thrombosis in liver cirrhosis. An angiographic study in 708 patients. Gastroenterology 89: 279-286, 1985.

3. Gaiani S, Bolondi L, Li BS, Zironi G, Siringo S, Barbara L. Prevalence of spontaneous hepatofugal portal flow in liver cirrhosis. Clinical and endoscopic correlation in 228 patients. Gastroenterology 100: 160-167, 1991.

4. Manzanet G, Sanjuan F, Orbis P. Liver transplantation in patients with portal vein thrombosis. Liver Transpl 7: 125-131, 2001.

5. Ogren M, Bergqvist D, Björck M, Acosta S, Eriksson H, Sternby NH. Portal vein thrombosis: prevalence, patient characteristics and lifetime risk: a population study based on 23,796 consecutive autopsies. World J Gastroenterol 12: 2115-2119, 2006.

6. Qi X, De Stefano V, Wang J. Prevalence of inherited antithrombin, protein $\mathrm{C}$, and protein $\mathrm{S}$ deficiencies in portal vein system thrombosis and Budd-Chiari syndrome: a systematic review and metaanalysis of observational studies. J Gastroenterol Hepatol 28: 432442, 2013.

7. Plessier A, Darwish-Murad S, Hernandez-Guerra M. Acute portal vein thrombosis unrelated to cirrhosis: a prospective multicenter follow-up study. Hepatology 51: 210-218, 2010.

8. De Stefano V, Finazzi G, Mannucci PM. Inherited thrombophilia: pathogenesis, clinical syndromes, and management. Blood 87: 3531-3544, 1996.

9. Melissari E, Monte G, Lindo VS, et al. Congenital thrombophilia among patients with venous thromboembolism. Blood Coagul Fibrinolysis 3: 749-758, 1992.

10. Picard V, Nowak-Göttl U, Biron-Andreani C, et al. Molecular bases of antithrombin deficiency: twenty-two novel mutations in the antithrombin gene. Hum Mutat 27: 600, 2006.

11. Rodeghiero F, Tosetto A. The epidemiology of inherited thrombo- 
philia: the VITA Project. Vicenza Thrombophilia and Atherosclerosis Project. Thromb Haemost 78: 636-640, 1997.

12. Tait RC, Walker ID, Perry DJ, et al. Prevalence of antithrombin deficiency in the healthy population. Br J Haematol 87: 106-112, 1994.

13. Lane DA, Caso R. Antithrombin: structure, genomic organization, function and inherited deficiency. Baillieres Clin Haematol 2: 961998, 1989.

14. Olds RJ, Lane DA, Caso R, et al. Antithrombin III Budapest: a single amino acid substitution (429Pro to Leu) in a region highly conserved in the serpin family. Blood 79: 1206-1212, 1992.

15. Khor B, Van Cott EM. Laboratory tests for antithrombin deficiency. Am J Hematol 85: 947-950, 2010.

16. Patnaik MM, Moll S. Inherited antithrombin deficiency: a review. Haemophilia 14: 1229-1239, 2008.

17. Hirsh J, Piovella F, Pini M. Congenital antithrombin III deficiency. Incidence and clinical features. Am J Med 87: 34S-38S, 1989.

18. Menache D, Grossman BJ, Jackson CM. Antithrombin III physiology, deficiency, and replacement therapy. Transfusion 32: 580-588, 1992.

19. Okuda K, Bando K, Okada M, Wada M, Misawa K. A case of mesenteric venous thromboembosis caused by congenital antithrombin deficiency. Jpn Gastroenterol Surg 44: 577-583, 2011.

20. Konkle BA, Bauer KA, Weinstein R, Greist A, Holmes HE, Bonfiglio J. Use of recombinant human antithrombin in patients with congenital antithrombin deficiency undergoing surgical procedures. Transfusion 43: 390-394, 2003.

21. Menache D, O'Malley JP, Schorr JB, et al. Evaluation of the safety, recovery, half-life, and clinical efficacy of antithrombin III (human) in patients with hereditary antithrombin III deficiency. Blood 75: 33-39, 1990.

22. van den Belt AG, Sanson BJ, Simioni P, Prandoni P, Büller HR, Girolami A, Prins MH. Recurrence of venous thromboembolism in patients with familial thrombophilia. Arch Intern Med 157: 22272232, 1997.

23. Fukuda T, Kamisato C, Honda Y, et al. Impact of antithrombin deficiency on efficacy of edoxaban and antithrombin-dependent anticoagulants, fondaparinux, enoxaparin, and heparin. Thromb Res 131: 540-546, 2013.

24. Minami K, Kunagai K, Sugai Y, Nakamura K, Naito S, Oshima S. Efficacy for Xa inhibitor for venous thromboembolism in a patient with antithrombin deficiency. Intern Med 57: 2025-2028, 2018.

25. Kawano H, Maemura K. Edoxaban was effective for the treatment of deep vein thrombosis and pulmonary thromboembolism in a cancer patient with antithrombin III deficiency. Intern Med 55: 3285-3289, 2016.

26. Lane DA, Olds RJ, Conard J, et al. Pleiotropic effects of antithrombin strand 1C substitution mutations. J Clin Invest 90: 2422-2433, 1992.

27. Miyata T, Sato Y, Ishikawa J, et al. Prevalence of genetic mutations in protein $\mathrm{S}$, protein $\mathrm{C}$ and antithrombin genes in Japanese patients with deep vein thrombosis. Thromb Res 124: 14-18, 2009.

The Internal Medicine is an Open Access journal distributed under the Creative Commons Attribution-NonCommercial-NoDerivatives 4.0 International License. To view the details of this license, please visit (https://creativecommons.org/licenses/ by-nc-nd/4.0/).

(C) 2019 The Japanese Society of Internal Medicine

Intern Med 58: 1733-1737, 2019 